\title{
Discrimination of Storage Time for Pork Using Metal Oxide Semiconductor (MOS)-Type E-Nose
}

\author{
Jun Wang, Xuezhen Hong, Yongwei Wang* and Bo Zhou \\ Department of Biosystems Engineering, Zhejiang University, \\ 268 Kaixuan Road, Hangzhou 310029, PR China \\ (Received May 7, 2012; accepted August 14, 2012)
}

Key words: electronic nose, pork, discrimination, storage time

An investigation was conducted to evaluate the capacity of an electronic metal oxide semiconductor (MOS)-type nose (e-nose) to classify pork samples with different storage times $(0-6 \mathrm{~d})$. The effects of the headspace-generation time and pork sample mass on the response of the e-nose were studied using multivariate analysis of variance and oneway analysis of variance, respectively. The results showed that the pork sample mass had the most significant effect on the e-nose sensor response, followed by the headspacegeneration time. The optimum parameters were $10 \mathrm{~g}$ of sample mass with $5 \mathrm{~min}$ of headspace-generation time in a $500 \mathrm{~mL}$ vial. After either principal component analysis or linear discriminant analysis, the results showed that the e-nose with the optimum parameters can accurately classify the pork samples stored for $0-6 \mathrm{~d}$. A method using a back propagation neural network was also performed, and $91.43 \%$ of the prediction set (with $92.86 \%$ of the training set) was classified correctly using this model.

\section{Introduction}

The main ingredients of meat are water, protein, fat and small amounts of carbohydrates. Owing to its high nutritional value and good taste, the consumer demand for meat has been increasing markedly during the last few decades. However, meat is highly susceptible to spoilage and contamination by microorganisms. During storage, the ingredients are decomposed by enzymes and bacteria, producing odor. The protein is decomposed into ammonia, hydrogen sulfide, ethyl mercaptan, etc. The fat is decomposed into aldehydes and aldehyde acids, which cause odor. The carbohydrates are decomposed into alcohols, ketones, aldehydes and carboxylic acid gases. ${ }^{(1)}$ The odor become more intense with the reduction of meat freshness or deterioration. Consumption

${ }^{*}$ Corresponding author: e-mail: wywzju@zju.edu.cn 
of spoiled meat can cause serious health hazards.(2) Thus, it is necessary for a rapid and accurate detection system to be developed for microbiologically spoiled or contaminated meat. ${ }^{(3,4)}$

At present, in the meat industry there are three main traditional methods of detecting meat freshness: sensory evaluation based on the texture, color, organization status, viscidity and odor of the meat, ${ }^{(5)}$ detection of the total volatile basic nitrogen (TVBN) ${ }_{,}^{(6)}$ and aerobic plate counts of meat samples using standard protocols. ${ }^{(7)}$ The first method is quick but subjective; it is very difficult to evaluate slight differences in the meat freshness during the initial stage of putrefaction by this method and it requires people with certain professional skills. The latter two methods are objective, but they are destructive, complicated and time-consuming. Moreover, they are only used to analyze one or two specialized components instead of giving complete information on the whole meat. Thus, these traditional methods are consequently unsuitable for on-line application in the meat industry.

An electronic nose (e-nose), also known as an artificial olfactory system, simulates biological functions to identify some simple or complex odors. ${ }^{(8,9)}$ A typical e-nose system contains a selective chemical sensor array, a signal processing subsystem and a pattern recognition subsystem. The sensors in the sensor array are sensitive to different substances. For example, some sensors can discern ammonia and some can discern aldehydes. Thus, the whole sensor array can discern complex odors. Instead of detecting one or two components of the substances, the e-nose obtains the complete information required for identification. In the last decade, a few researchers have been trying to study the potential of using the e-nose as a nondestructive method for food detection. García et al..$^{(10)}$ used an e-nose based on metal oxide semiconductor (MOS) thin-film sensors to characterize and classify four types of red wines made from the same variety of grapes which came from the same cellar. Two pattern recognition methods, principal component analysis (PCA) and a probabilistic neuronal network (PNN) were used and the results showed that the e-nose was able to identify the wines well. Torri et al. ${ }^{(11)}$ used a commercial e-nose to monitor the freshness of minimally processed fruit (packaged pineapple slices) during storage. The samples were stored at three different temperatures $\left(4-5,7-8\right.$, and $\left.15-16^{\circ} \mathrm{C}\right)$ for $6-10 \mathrm{~d}$. After continuous monitoring of the headspace around the fruit, the result showed that the fruit freshness was maintained for about $5 \mathrm{~d}$ at $4{ }^{\circ} \mathrm{C}, 2 \mathrm{~d}$ at $7.6^{\circ} \mathrm{C}$ and $1 \mathrm{~d}$ at $16^{\circ} \mathrm{C}$.

However, studies using the e-nose are in their infancy and the recognition models used often only focused on discrimination. For example, Hansen et al. ${ }^{(12)}$ in Denmark investigated the sensory quality of pork meatloaf using an electronic nose with six MOS sensors. A multivariate data analysis strategy (involving analysis of variance, partial least-squares regression, and PCA) was used to determine causal and predictive relationships between the raw material and sensory analysis results obtained by the electric nose. The results showed that the six MOS sensors in the odor sensor system could detect the raw materials that led to unacceptable products, as determined by sensory profiling and in-house sensory quality control. Vestergaard et al. ${ }^{(13)}$ investigated the predictability of an electronic nose system based on ion mobility regarding sensory quality changes during the storage of a pork meat pizza topping product and found the 
electronic nose system to be a relevant device for future at- or on-line implementation in the quality control of the pork meat pizza topping product.

Moreover, few papers have reported the study of optimum experimental parameters and there has been little research on the discrimination of pork storage time using an e-nose. The main objective of this research is to evaluate the capacity of an e-nose to classify pork samples with different storage times $(0-6 \mathrm{~d})$. The effects of headspacegeneration time and pork sample mass on the response of the e-nose were studied and the optimum experimental parameters of headspace-generation time and sample mass were determined after employing multivariate analysis of variance (MANOVA) and one-way analysis of variance (ANOVA), respectively. The latter experiment was carried out with the optimum experimental parameters. Three methods, PCA, linear discriminant analysis (LDA) and a back propagation neural network (BPNN), were employed to determine if the e-nose can accurately classify the pork samples stored for $0-6 \mathrm{~d}$, as well as predict the storage time of the meat samples.

\section{Materials and Method}

\subsection{Sample preparation}

Fresh lean pork samples were purchased twice, the first time $(1750 \mathrm{~g})$ was for the preliminary experiment to obtain the optimum parameters and the second time $(1100 \mathrm{~g})$ was for the discrimination of pork samples stored for $0-6 \mathrm{~d}$. Both samples were obtained from the same parts of pigs and the same supplier in the local farmers' market $(30.26$ $\mathrm{N}, 120.19 \mathrm{E}$, Zhejiang province, China) $4 \mathrm{~h}$ after being slaughtered and were minced immediately on the spot. All the samples were packed immediately using polystyrene base trays and were covered with commercial food-grade polymer wraps just before being transported to the lab. The samples were stored at $5^{\circ} \mathrm{C}$ in a fridge before detection, except for the ones examined on the first day (denoted as day 0 ).

\subsection{Electronic nose system}

The experiment was performed with a portable electronic nose (PEN2, Airsense Analytics, GmBH, Schwerin, Germany) (Fig. 1). This instrument consists of an autosampling apparatus that is exposed to the volatile gases, an array of sensors and pattern recognition software that is run on a computer. The sensor array is composed of ten different MOSs. A description of the ten MOSs is given in Table 1.

\subsection{Experimental procedure}

The concentration of the volatile gases was affected by the mass and headspacegeneration time of the samples, ${ }^{(14)}$ so a set of preliminary experiments were performed to determine the optimum experimental parameters. Three main factors were considered: (1) mass of the pork samples (M: 10 and $25 \mathrm{~g}$ ); (2) storage time (ST: 0 and $1 \mathrm{~d}$ ); and (3) headspace-generation time (HGT: 5, 15, and $25 \mathrm{~min}$ ). The samples stored for 0 and $1 \mathrm{~d}$ were each divided into six groups, denoted as $10-5,10-15,10-25,25-5,25-15$, $25-25$. The number format is mass - headspace-generation time, for example, the $10-$ 5 group means $10 \mathrm{~g}$ of sample with 5 min of headspace-generation time. The multifactor 


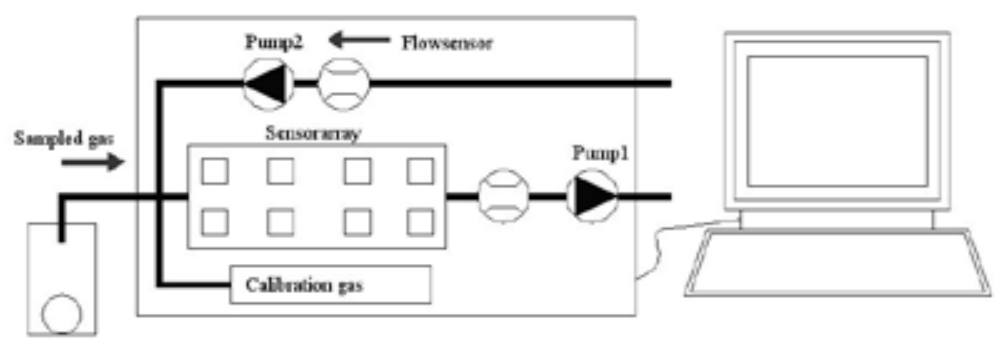

Fig. 1. Structure of e-nose.

Table 1

Sensors used and their object substances in PEN2.

\begin{tabular}{lll}
\hline Array number & Sensor & Substances for sensing \\
\hline MOS 1 & W1C & Aromatics \\
MOS 2 & W5S & Nitrogen oxides \\
MOS 3 & W3C & Ammonia, aromatic molecules \\
MOS 4 & W6S & Hydrogen \\
MOS 5 & W5C & Methane, propane and aliphatic nonpolar molecules \\
MOS 6 & W1S & Methane \\
MOS 7 & W1W & Sulfur-containing organics \\
MOS 8 & W2S & General alcohols \\
MOS 9 & W2W & Aromatics, sulfur- and chlorine-containing organics \\
MOS 10 & W3S & Methane and aliphatics \\
\hline
\end{tabular}

preliminary experiment was conducted with seven replicates for each group (Table 2). After determining the optimum experimental parameters, the pork samples stored for $0-6 \mathrm{~d}$ ( 15 replicates for each day) were detected using the e-nose under these parameters.

Each pork sample was placed in a $500 \mathrm{~mL}$ airtight glass vial that was sealed with a plastic wrap. The glass vial was closed for a certain time (headspace-generation time) while the headspace collected the volatile gases from the pork sample. During the measurement, the headspace gases were pumped into the sensor arrays through Teflon tubing connected to a needle in the plastic wrap. The measurement phase lasted for $65 \mathrm{~s}$, which was long enough for the sensors to reach stable signal values. The signal data from the sensors were collected with a computer once every second during the measurements. When the measurement was complete, the acquired data were stored for later use. The experiment and measurements were carried out at $20 \pm 1{ }^{\circ} \mathrm{C}$. After each experiment, zero gas (air filtered with active carbon) was pumped into the sample gas path from the other port of the instrument. 
Table 2

Preliminary experiment method.

\begin{tabular}{lccc}
\hline $\begin{array}{l}\text { Storage } \\
\text { time (d) }\end{array}$ & Mass (g) & $\begin{array}{c}\text { Headspace-generation } \\
\text { time (min) }\end{array}$ & $\begin{array}{c}\text { Number of } \\
\text { samples }\end{array}$ \\
\hline 0 & 15 & 5 & 7 \\
0 & 15 & 15 & 7 \\
0 & 15 & 25 & 7 \\
0 & 25 & 5 & 7 \\
0 & 25 & 15 & 7 \\
0 & 25 & 25 & 7 \\
1 & 15 & 5 & 7 \\
1 & 15 & 15 & 7 \\
1 & 15 & 25 & 7 \\
1 & 25 & 5 & 7 \\
1 & 25 & 15 & 7 \\
1 & 25 & 25 & 7 \\
\hline
\end{tabular}

\subsection{Statistical analysis}

PCA is a very powerful multivariate statistical method used to analyze the inherent structure of data. ${ }^{(15)}$ The main purpose of this linear feature extraction method is to reduce dimensions by projecting the $m$-dimensional data set ( $m$ is the number of sensors) into a dimension smaller than $m$. These new variables are more descriptive because they are chosen to obtain the maximum variance in the data matrix, ${ }^{(16)}$ and based on them, the samples differ from each other. Loadings represent coefficients of the principal components (PCs) and indicate how much the original variables contribute to the PCs. Scores reflect the location of the samples along the PCs and show sample differences or similarities. ${ }^{(17)}$

LDA is another widely used statistical method. Similar to PCA, it also takes a linear combination of the original variables to construct a discriminant function. ${ }^{(18)}$ Compared with PCA, the LDA method can be used to determine the distribution of points in the same category and the distance between them. It maximizes the variance between categories and minimizes the variance within categories to improve the resolution of classes. ${ }^{(19)}$ The graphical view of LDA results is similar to a PCA display.

The BPNN has been a widely used method for the e-nose. ${ }^{(20-22)}$ The BPNN can be described as a nonlinear projection between the input vectors and output vectors. A typical BPNN model includes an input layer, a hidden layer (one layer or more), and an output layer.

Three eigenvalues (maximum, average and the $60 \mathrm{~s}$ value of the sensor signal) of every sensor signal were adopted and then all the 30 data from the ten sensor signal values were used as the input vector of the BPNN. The output vector was designed to be seven-dimensional, in accordance with the seven storage days. The training goal error was 0.001 . In accordance with the dimensions of the input and output vectors, as 
well as many trials, the network topology was a four-layer net designed as 30-7-7-7. While determining the suitable network topology, the network processes the inputs and compares the resulting outputs against the desired outputs. Errors are then propagated back through the system, causing the system to adjust the weights that control the network. ${ }^{(15)}$ This process occurs over and over until the error matches the training goal error.

The data processing method, PCA, LDA and ANOVA were performed using SAS software and the BPNN was operated using the network toolbox in MATLAB R2008a.

\section{Results and Discussion}

\subsection{Response signal of sensors}

Figure 2 shows typical response curves of the ten sensors during the measurement of a pork sample. Each curve represents a sensor's ratio of conductance $\left(G / G_{0}\right.$, where $G$ and $G_{0}$ are the conductivities of the sensor when exposed to the sample gas and zero gas, respectively). At first, the conductivities of sensors MOS1 and MOS3 increased quickly, whereas those of sensors MOS2, MOS7, MOS8, and MOS9 decreased quickly, those of sensors MOS5, MOS4, and MOS6 changed slowly, and that of sensor MOS10 almost remained the same. Finally, all the sensors stabilized after about $55 \mathrm{~s}$. Thus, to save time, the measurement time was set at $65 \mathrm{~s}$ and the flush time was set at $50 \mathrm{~s}$ in this research. The response values of each sensor at $60 \mathrm{~s}$ were extracted for further analysis.

\subsection{Optimum parameters}

\subsubsection{MANOVA result}

A 3-factor analysis of variance was performed to see how these factors affected the response of the e-nose. The three factors are $M$ (sample mass), $T$ (headspace-generation time) and $D$ (storage time). The statistical analysis results are summarized in Table 3. The magnitudes of the $F$-values indicate the relative importance of the factors to some

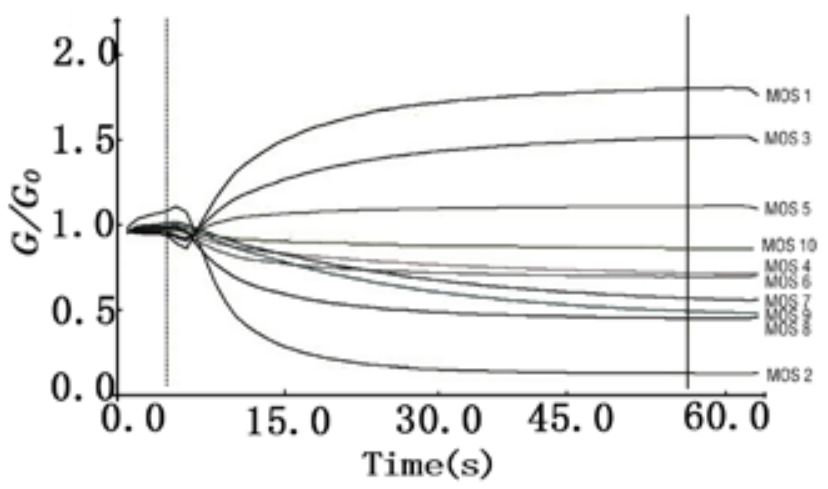

Fig. 2. Response curves of sensors for pork sample. 
Table 3

MANOVA results (factors are storage days, mass and headspace-generation time).

\begin{tabular}{lrcrrc}
\hline DS & \multicolumn{1}{c}{$S S$} & $D F$ & \multicolumn{1}{c}{$M S$} & \multicolumn{1}{c}{$F$} & $p$ \\
\hline$M$ & 17.880 & 1 & 17.880 & 172.231 & 0.000 \\
$T$ & 8.043 & 2 & 4.021 & 38.736 & 0.000 \\
$D$ & 22.875 & 1 & 22.875 & 220.338 & 0.000 \\
$M \times T$ & 0.890 & 2 & 0.445 & 4.288 & 0.017 \\
$M \times D$ & 0.145 & 1 & 0.145 & 1.393 & 0.241 \\
$T \times D$ & 0.217 & 2 & 0.108 & 1.044 & 0.357 \\
$M \times T \times D$ & 0.678 & 2 & 0.339 & 3.267 & 0.043 \\
\hline
\end{tabular}

$D S$ : different source, $S S$ : sum of squares, $D F$ : degree of freedom, $M S$ : mean squares.

extent.

As shown in Table 3, the mass of the sample, the storage time and the headspacegeneration time all had a very significant effect on the response of the e-nose $(p<0.001)$. The interactions of $M \times T$ and $M \times T \times D$ also had a significant effect on the response of the e-nose $(p<0.05)$. It can also be observed that the storage time had the highest $F$-value, which means that the e-nose signals differed between samples with different storage times. This proved the feasibility of using the e-nose to distinguish pork with different storage times. The mass factor had the second highest $F$-value, followed by the headspace-generation time. Thus, it is very important to determine the sample mass and headspace-generation time.

\subsubsection{ANOVA result}

ANOVA was carried out for groups $10-5,10-15,10-25,25-5,25-15$, and $25-25$ and the $F$-value for each group was obtained. The results of the six groups are summarized in Table 4.

As shown in Table 4, all the groups had very significant effects on the response of the e-nose and the combination $10-5$ had the highest $F$-value. This means that when the mass is $10 \mathrm{~g}$ and the headspace-generation time is $5 \mathrm{~min}$, the e-nose has the most obvious difference between samples with different storage times. Therefore, in this research, the optimum parameters are $10 \mathrm{~g}$ of pork sample with $5 \mathrm{~min}$ of headspace-generation time.

\subsubsection{Discrimination power (DP)}

DP is another index used to observe the magnitude of difference among the samples. ${ }^{(22)}$ All the combinations with different masses and headspace-generation times (as described before) were subjected to DP analysis and a DP value was obtained (listed in Table 5). The number format is storage time - mass - headspace-generation time; for example, $0-10-5$ means stored for 0 day, $10 \mathrm{~g}$ of sample mass with $5 \mathrm{~min}$ of headspace-generation time. As shown in Table 5, the $10-5$ combination has the highest DP value, which means that the e-nose response of this combination has the most obvious difference between day 0 and day 1 . This result is the same as the ANOVA result. 
Table 4

$F$-values of six combinations.

\begin{tabular}{lrr}
\hline Combinations & $F$-value & $P$ \\
\hline $10-5$ & 209.883 & 0.000 \\
$10-15$ & 44.131 & 0.000 \\
$10-25$ & 66.676 & 0.000 \\
$25-5$ & 89.451 & 0.000 \\
$25-15$ & 159.226 & 0.000 \\
$25-25$ & 55.049 & 0.000 \\
\hline
\end{tabular}

The number format is mass - headspace-generation time; for example, $10-5$ means $10 \mathrm{~g}$ of sample with 5 min of headspace-generation time.

Table 5

Results of DP test of six combinations.

\begin{tabular}{|c|c|c|c|c|c|c|}
\hline & $1-10-5$ & $1-10-15$ & $1-10-25$ & $1-25-5$ & $1-25-15$ & $1-25-25$ \\
\hline $0-10-5$ & 0.967 & & & & & \\
\hline $0-10-15$ & & 0.555 & & & & \\
\hline $0-10-25$ & & & 0.753 & & & \\
\hline $0-25-5$ & & & & 0.582 & & \\
\hline $0-25-15$ & & & & & 0.852 & \\
\hline $0-25-25$ & & & & & & 0.448 \\
\hline
\end{tabular}

The number format is storage time - mass - headspace-generation time: for example, 0 - 10-5 means stored for 0 day, $10 \mathrm{~g}$ of sample with $5 \mathrm{~min}$ of headspace-generation time.

\section{$3.3 \quad P C A$}

The pork samples stored for $0-6 \mathrm{~d}$ were detected using the e-nose under optimum experimental parameters. For all the pork samples, the response signals of the e-nose at $60 \mathrm{~s}$ were extracted and analyzed by PCA. The results are shown in Fig. 3, which is a two-dimensional spatial PCA plot defined by the first two principal components. The first principal component (PC 1) provides $94.15 \%$ of the total variance and the second principal component (PC 2) provides $3.60 \%$ of the total variance; the total contribution rate is $97.75 \%$, which means that these two components reflect $97.75 \%$ of the original information. As seen from the score plot in the area defined using the first two principal components, the samples stored for $0-3 \mathrm{~d}$ are very concentrated and the samples stored for 1-3 $\mathrm{d}$ are very close to each other. This may be explained as follows: for the first $1-$ $3 \mathrm{~d}$, fresh pork stored at $5^{\circ} \mathrm{C}$ in the fridge still remained fresh; the change in the volatile gases of pork was subtle so the e-nose could not notice the difference. Thus, the data extracted by the e-nose were similar. However, freezing and unfreezing could cause cell damage and affect the quality of pork. Thus, the samples stored for $0 \mathrm{~d}$, which were used for the experiment directly without being stored in the fridge, were discriminated from those stored in the fridge for 1-3 d. In general, all the samples can be clearly divided into seven regions according to their storage time, except for overlapping for the samples stored for $2-3 \mathrm{~d}$. 


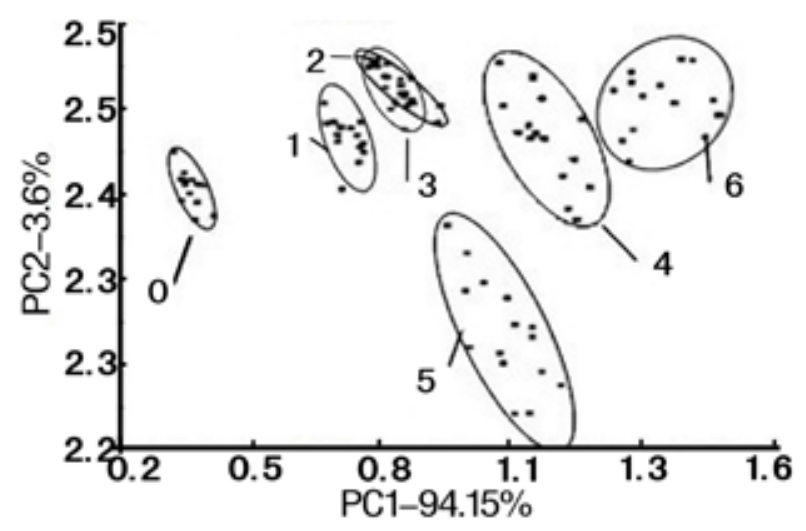

Fig. 3. PCA analysis of meat stored from $0-6 \mathrm{~d}$.

\section{$3.4 L D A$}

The same data analyzed by PCA were extracted by LDA (Fig. 4). The first discriminant function (LD1) provides $64.10 \%$ of the total variance and the second discriminant function (LD2) provides $20.24 \%$ of the total variance; the total contribution rate is $84.34 \%$. Similar to the PCA results, although the samples stored for $2 \mathrm{~d}$ have an overlap with the samples stored for $3 \mathrm{~d}$, the samples can be clearly divided into seven regions according to their storage time.

\section{$3.5 \quad B P N N$}

In this study, a 30-7-7-7 BPNN model was used for the storage time prediction of the pork samples stored for $0-6 \mathrm{~d}$. The 105 samples ( 7 storage times $\times 15$ duplicates) were divided into two groups: 70 samples for the training set $(10$ samples from each group were randomly chosen) and 35 samples for the prediction set. Thus, the input layers for the training set and prediction set were a $70 \times 30$ matrix and a $35 \times 0$ matrix, respectively. The results are shown in Tables 6 and 7, respectively.

The total identification rate of the simulation results for the training set was $93.33 \%$ and the total identification rate for the prediction set was $92.38 \%$. It should be noticed that neither the prediction set nor the training set could correctly discriminate the samples stored between 2 and $3 \mathrm{~d}$. This result is in accordance with the results obtained by PCA and LDA; this implied that the pork samples stored for the first 2 or $3 \mathrm{~d}$ changed little and remained fresh. 


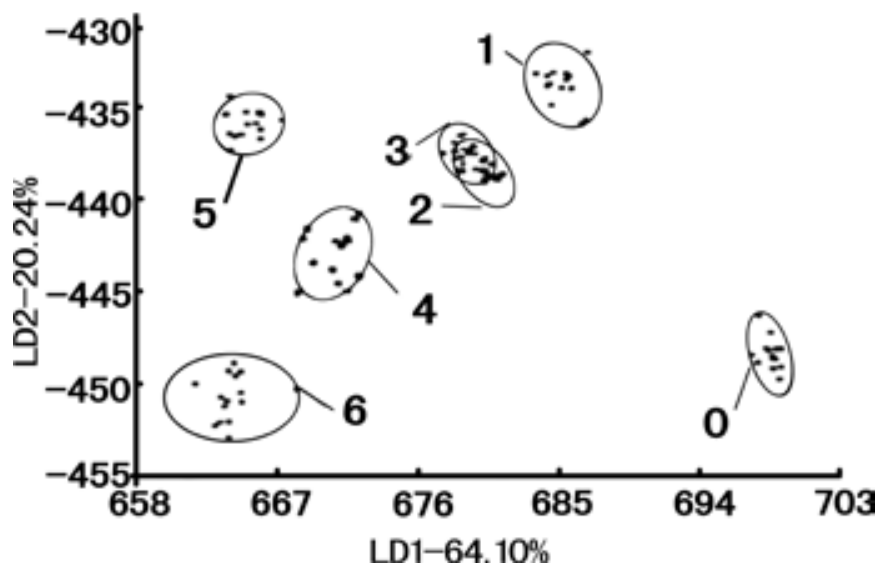

Fig. 4. LDA analysis of meat stored from 0-6 d.

Table 6

$\mathrm{BP}$ results for the original data in the training set.

\begin{tabular}{|c|c|c|c|c|c|c|c|c|c|c|}
\hline \multirow[t]{2}{*}{$\overline{S T}$} & \multirow[t]{2}{*}{$N S$} & \multicolumn{7}{|c|}{ Recognition results } & \multirow{2}{*}{$\begin{array}{c}\text { Identification rate of } \\
\text { each day }\end{array}$} & \multirow{2}{*}{$\begin{array}{l}\text { Identification } \\
\text { rate of all days }\end{array}$} \\
\hline & & 0 & 1 & 2 & 3 & 4 & 5 & 6 & & \\
\hline$\overline{0}$ & 10 & 10 & & & & & & & $100 \%$ & \\
\hline 1 & 10 & & 10 & & & & & & $100 \%$ & \\
\hline 2 & 10 & & & 7 & 3 & & & & $70 \%$ & \\
\hline 3 & 10 & & & & 9 & 1 & & & $90 \%$ & $92.86 \%$ \\
\hline 4 & 10 & & & & & 10 & & & $100 \%$ & \\
\hline 5 & 10 & & & & & & 9 & 1 & $90 \%$ & \\
\hline 6 & 10 & & & & & & & 10 & $100 \%$ & \\
\hline
\end{tabular}

$S T$ : storage time; $N S$ : number of samples

Table 7

$\mathrm{BP}$ results for the original data in the prediction set.

\begin{tabular}{|c|c|c|c|c|c|c|c|c|c|c|}
\hline \multirow[t]{2}{*}{$\overline{S T}$} & \multirow[t]{2}{*}{$N S$} & \multicolumn{7}{|c|}{ Recognition results } & \multirow{2}{*}{$\begin{array}{c}\text { Identification rate of } \\
\text { each day }\end{array}$} & \multirow{2}{*}{$\begin{array}{l}\text { Identification } \\
\text { rate of all days }\end{array}$} \\
\hline & & 0 & 1 & 2 & 3 & 4 & 5 & 6 & & \\
\hline$\overline{0}$ & 5 & 5 & & & & & & & $100 \%$ & \\
\hline 1 & 5 & & 5 & & & & & & $100 \%$ & \\
\hline 2 & 5 & & & 2 & 3 & & & & $40 \%$ & \\
\hline 3 & 5 & & & & 5 & & & & $100 \%$ & $91.43 \%$ \\
\hline 4 & 15 & & & & & 5 & & & $100 \%$ & \\
\hline 5 & 5 & & & & & & 5 & & $100 \%$ & \\
\hline 6 & 5 & & & & & & & 5 & $100 \%$ & \\
\hline
\end{tabular}




\section{Conclusions}

(1) The result of MANOVA shows that it is feasible to discriminate pork with different storage times using the MOS-type e-nose. The sample mass and headspacegeneration time have a significant effect on the response of the e-nose. By ANOVA and DP analysis, the optimum experimental parameters were determined to be $10 \mathrm{~g}$ of sample mass with 5 min of headspace-generation time.

(2) The storage time of pork can be discriminated by PCA or LDA. The samples can be clearly divided into seven regions according to their storage time, except for overlapping between the samples stored for 2 and $3 \mathrm{~d}$. The LDA method was superior to PCA for the discrimination of the pork samples based on the MOS-type e-nose signals.

(3) A 30-7-7-7 BPNN model was used for the prediction of the storage time. The results showed that $92.38 \%$ of the prediction set and $93.33 \%$ of the training set were classified correctly using this model. The incorrect discrimination mainly came from the confusion between the samples stored for 2 and $3 \mathrm{~d}$. This implied that the pork samples stored for the first 2 or $3 \mathrm{~d}$ changed little and remained fresh.

\section{Acknowledgements}

The authors acknowledge the financial support of the Chinese National Foundation of Nature and Science through Project 31071548 and the Research Fund for the Doctoral Program of Chinese National Higher Education through Project 20100101110133.

\section{References}

1 B. H. Kong and L. Z. Ma: Meat Science and Technology (Chinese Light Industry Press, Beijing, 2003) pp. 97-99.

2 L. Gram, L. Ravn, M. Rasch, J. B. Bruhn, A. B. Christensen and M. Givskov: Int. J. Food Microbiol. 78 (2002) 79.

3 D. L. Archer: Food Control 7 (1996) 3.

4 D. I. Ellis, D. Broadhurst and R. Goodacre: Anal. Chim. Acta 514 (2004) 193.

5 N. Shirsat, N. P. Brunton, J. G. Lyng, B. McKenna and A. Scannell: J. Sci. Food Agric. 84 (2004) 1861.

6 P. Malle, A. M. Vanelle and A. Petit: Rec. Med. Vet. 165 (1989) 395.

7 FDA-US Food \& Drugs Administration: Bacteriological Analytical Manual, 8th ed. (AOAC International, Gaithersburg, MD, 1998).

8 H. C. Yu, J. Wang, C. Yao, H. Zhang and Y. Yu: LWT Food Sci. Technol. 41 (2008) 1268.

9 H. Zhang, M. Chang, J. Wang and S. Ye: Sens. Actuators, B 134 (2008) 332.

10 M. García, M. Alexandre, J. Gutiérrez and M. C. Horrillo: Sens. Actuators, B 113 (2006) 911.

11 L. Torri, N. Sinelli and S. Limbo: Postharvest Biol. Technol. 56 (2010) 239.

12 T. Hansen, M. A. Petersen and D. V. Byrne: Meat Sci. 69 (2005) 621.

13 J. S. Vestergaard, M. Martens and P. Turkki: LWT Food Sci. Technol. 40 (2007) 1095.

14 J. Olsson, T. Börjesson, T. Lundstedt and J. Schnürer: Int. J. Food Microbiol. 72 (2002) 203.

15 L. J. Pang, J. Wang, X. Lu and H. Yu: Trans. ASABE 51 (2008) 1707.

16 S. Capone, P. Siciliano, F. Quaranta, R. Rella, M. Epifani and L. Vasanelli: Sens. Actuators, B 69 (2000) 230. 
17 C. Benfeldt and J. Sorensen: Int. Dairy J. 11 (2001) 567.

18 S. Buratti, S. Benedetti, M. Scampicchio and E. C. Pangerod: Anal. Chim. Acta 525 (2004) 133.

19 K. Neely, C. Taylor, O. Prosser and P. F. Hamlyn: Meat Sci. 58 (2001) 53.

20 N. Magana and P. Evans: J. Stored Prod. Res. 36 (2000) 319.

21 Q. Y. Zhang, S. P. Zhang, C. S. Xie, D. W. Zeng, C. Q. Fan and D. F. Li: Sens. Actuators, B 119 (2006) 538.

22 H. Yu, J. Wang, H. Zhang, Y. Yu and C. Yao: Sens. Actuators, B 128 (2008) 455. 